\title{
Distribución y estado de conservación de las poblaciones de árboles y arbustos del género Polylepis (Rosaceae) en las montañas de Argentina
}

\author{
Daniel Renison ${ }^{1,2, 凶}$; Griet A.E. Cuyckens ${ }^{3}$; Silvia PacheCo ${ }^{4}$; Gustavo F. Guzmán ${ }^{5}$; H. \\ Ricardo Grau ${ }^{6}$; Paula Marcora ${ }^{2,7}$; Gerardo Robledo ${ }^{7}$; Ana M. Cingolani ${ }^{2,7}$; Julio \\ Dominguez ${ }^{2}$; Marcos LANDi ${ }^{8}$; LAURA Bellis ${ }^{8} \&$ ISABEll Hensen ${ }^{9}$
}

1. Centro de Ecología y Recursos Naturales Renovables Dr. Ricardo Luti (CERNAR-IIByT), FCEFyN (UNC-CONICET). Córdoba, Argentina. 2. Ecosistemas Argentinos, Asociación Civil. 3. Cátedra de Ecología de Comunidades, Facultad de Ciencias Agrarias (UNJu-CONICET). 4. Fundación ProYungas. 5. Cátedra de Ecología Agrícola, Facultad de Ciencias Agrarias (UNJu). 6. Instituto de Ecología Regional (UNT-CONICET). 7. Instituto Multidisciplinario de Biología Vegetal (CONICETUNC). 8. Instituto de Diversidad y Ecología Animal (CONICET-UNC). 9. Institute of Biology/Geobotany and Botanical Garden, Martin Luther University

\begin{abstract}
Resumen. El género Polylepis es endémico de las montañas de Sudamérica. Sus especies frecuentemente dominan la canopia de bosques y arbustales cuya conservación y restauración son una prioridad a nivel continental. En Argentina, el género incluye cinco especies que se distribuyen en las montañas del centro y noroeste del país. Sus distribuciones, amenazas y estados de conservación están poco documentados e incluso existen fuertes controversias sobre el grado en que el Hombre ha impactado en la cobertura, la estructura y la distribución de estos bosques. Para contribuir a revertir esta situación confeccionamos modelos potenciales por especie que sugieren $14800 \mathrm{~km}^{2}$ aptos para la presencia de al menos una de las cinco especies. Evaluamos 490 parcelas de $3600 \mathrm{~m}^{2}$ distribuidas en 43 localidades y para cada especie describimos los bosques en mejor estado de conservación que de manera tentativa podrían usarse como referencia para determinar metas de conservación y en trabajos de restauración. En tres de las cinco especies encontramos evidencias de fuego en más de $60 \%$ de las parcelas visitadas. Muchas localidades presentaban suelos con evidencias de erosión y escasa cobertura de vegetación, así como una baja cobertura y complejidad estructural de bosque. Consideramos que Argentina ofrece buenas oportunidades para la conservación y restauración de estos bosques ya que aún quedan grandes extensiones, numerosos relictos, y en muchas áreas la presión antrópica ha disminuido. Es prioritario reducir los fuegos, establecer más y mejores áreas protegidas y, en particular en el noroeste Argentino, tener un mejor conocimiento de la ecología de cada especie y desarrollar métodos para restaurar sus bosques.
\end{abstract}

[Palabras clave: Modelos de distribución, MaxEnt, amenazas, bosques, bosquecillos]

\begin{abstract}
Aвstract. Distribution and conservation status of the trees and shrubs of the Polylepis genus (Rosaceae) in the mountains of Argentina: The genus Polylepis is endemic to the mountains of South America. Species of this genus frequently dominate the canopy of forests and shrublands whose conservation and restoration is a priority at a continental scale. The five species described for Argentina are distributed in the mountains central and northwestern Argentina. Their distribution, threats and conservation status are poorly described and the degree to which humans have impacted their cover, structure and distribution is controversial. We modeled the distribution per species. Models suggest an area of $14800 \mathrm{~km}^{2}$ environmentally suitable for at least one of the five species. We surveyed 490 plots of $3600 \mathrm{~m}^{2}$ distributed in 43 locations and for each species we describe the best preserved forests which could tentatively be used as reference sites when determining conservation and restoration goals. In three of the species we recorded fire evidences in over $60 \%$ of the visited plots. Soil erosion was evident in several localities and many forests presented low cover and structural complexity. Argentina provides good opportunities for the conservation and restoration of these forests as there still remain extensive forests, numerous relicts, and in many areas human impacts have been decreasing. It is important to reduce fire events, establish more and better protected areas, to have a better knowledge on the ecology of each species and to develop methods to restore forests, especially in northwestern Argentina.
\end{abstract}

[Keywords: Distribution models, MaxEnt, threats, forests, woodlands]

\section{INTRODUCCIÓN}

Los árboles y arbustos del género Polylepis (familia de las Rosáceas, comúnmente llamados "queñoa" o "tabaquillo") son endémicos de las montañas de Sudamérica.

$\triangle$ Centro de Ecología y Recursos Naturales Renovables Dr. Ricardo Luti (CERNAR-IIByT), FCEFyN (UNCCONICET). Córdoba, Argentina.

drenison@conicet.gov.ar
Sus especies a menudo dominan la canopia de bosques y arbustales de montaña (de aquí en más "bosques de Polylepis") desde la cordillera de Mérida (Venezuela) hasta las serranías del centro Argentino (Simpson 1979; Kessler \& Schmidt-Lebuhn 2006). 
Según estudios polínicos realizados en Bolivia y Perú, los bosques de Polylepis nunca formaron una masa forestal continua, más bien mosaicos de pastizales y bosquecillos de tamaños diversos que muestran una sensibilidad elevada a los cambios climáticos. La tendencia desde las últimas glaciaciones hasta la actualidad ha sido de una notable reducción en los registros polínicos (Gosling et al. 2009). Algunos estudios realizados en una escala temporal más actual registran una reducción de la superficie de bosques, además de una reducción en su densidad y complejidad estructural (Fjeldså \& Kessler 2004; Purcell \& Brisford 2004; Braun Wilke et al. 2005; Renison et al. 2011). Las causas de dicha reducción son controvertidas, en parte porque difieren entre regiones y entre especies de Polylepis. Además, es difícil separar los efectos del cambio climático desde las últimas glaciaciones con los efectos que pudiera haber tenido el humano sobre los bosques de Polylepis. Esto es debido a que los primeros humanos comenzaron a habitar estas regiones poco después de la finalización de las glaciaciones, y existe la posibilidad de que incluso unos pocos habitantes, ayudados con el uso del fuego, podrían incidir fuertemente en la distribución y la estructura de los bosques de Polylepis. Esto es muy evidente desde hace alrededor de 4000 años (Paduano et al. 2003). Más allá de las controversias, se sabe que el fuego, el pastoreo por ganado doméstico, la tala y la agricultura tienen un papel importante en la declinación de los bosques de Polylepis (Kessler 2000). Por ello, y debido a la elevada diversidad de endemismos asociada a estos bosques, se considera a nivel continental que su conservación y restauración es una prioridad (Fjeldså \& Kessler 2004).

En todos los países donde existen bosques de Polylepis se están realizando esfuerzos para su conservación y restauración, desde la creación de áreas protegidas, investigación, hasta numerosos proyectos de reforestación. Aún así es mucho lo que falta. A modo de ejemplo, en casi todas las especies de Polylepis resta mapear su distribución completa (a excepción de las especies de Bolivia incluidas en Navarro et al. 2010). Se desconoce, además, cuál sería la extensión, la estructura y la diversidad biológica de los bosques de Polylepis sin una intervención humana fuerte. Esta información es importante ya que podría ser tomada para diseñar áreas de referencia a tener en cuenta a la hora de tomar decisiones de manejo (SER 2004).

Hasta el momento, en Argentina se han identificado cinco especies de Polylepis: $P$. tomentella, $P$. australis, $P$. hieronymi (Simpson 1979), P. tarapacana (Morales et al. 2004) y $P$. crista-galli (Kessler \& Schmidt-Lebuhn 2006). En las últimas décadas se avanzó de manera importante en el estudio de la ecología y conservación de algunas de estas especies, y ya existen al menos 62 publicaciones relacionadas al tema (Información Suplementaria, Apéndice 1). Sin embargo, no existen mapas de distribución completos para ninguna de las especies, y hasta el momento, $80 \%$ de los estudios están concentrados en el sector sur de distribución del género (Sierras de Córdoba), donde encontramos sólo a P. australis.

En Argentina, la ganadería es la principal actividad económica en las montañas donde se encuentran los bosques de Polylepis. Según estudios realizados en el las Sierras de Córdoba, la ganadería estimula de manera leve la regeneración temprana (Zimmermann et al. 2009) pero perjudica por ramoneo a los juveniles con altura de 4 a $150 \mathrm{~cm}$, que cada año pierden hasta $98 \%$ de la biomasa que producen (Giorgis et al. 2010). Típicamente, la ganadería se asocia a la quema de pastizales y bosques, lo cual restringe a los individuos de $P$. australis a los sitios menos combustibles (e.g., posiciones topográficas bajas y sitios rocosos) (Renison et al. 2002; 2006). En consecuencia, cientos de años de ganadería doméstica y fuegos, junto con la tala, aparentemente han producido un marcado decrecimiento del área de distribución de $P$. australis en las Sierras de Córdoba (Cingolani et al. 2008), así como un aumento en la erosión de los suelos, una simplificación de la estructura vertical del bosque y una disminución de la biodiversidad que albergan (Bellis et al. 2009; Renison et al. 2010, 2011; Robledo \& Renison 2010).

Los hallazgos realizados en $P$. australis de las Sierras de Córdoba son difíciles de extrapolar a las otras regiones ya que las distintas regiones y especies de Polylepis pueden ser muy diferentes en cuanto a su ecología e historia de uso. A modo de ejemplo, en Córdoba se menciona a la ganadería y a los fuegos como los principales disturbios que afectan a los bosques. Sin embargo, no hay reportes de que el ganado se alimente de ninguna de las otras especies de Polylepis. Por otro lado, los únicos dos estudios de rebrote post-fuego sugieren, sobre la base de observaciones de un único fuego por región, una capacidad alta de rebrote para P. australis en Córdoba (Renison et al. 2002) y nula para $P$. incana en Ecuador (Cierjacks et al. 2008). La agricultura se menciona como uno de los disturbios importantes en bosques de Polylepis de Bolivia 
(Fjeldså \& Kessler 2004) pero sin mención para Argentina.

Dados los reportes acerca de la reducción de la superficie de los bosques de Polylepis y la falta de información sobre su distribución y estado de conservación, este trabajo tuvo tres objetivos: 1) modelar el área de distribución potencial de las especies de Polylepis en base a características ambientales, 2) evaluar cuáles son las amenazas a sus bosques, el estadodeconservación y las posibles áreas dereferencia mediante relevamientos rápidos de campo, y 3) discutir estrategias generales de conservación y líneas de investigación prioritarias. El trabajo es parte de un esfuerzo mayor queintenta plasmar estos objetivos en todos los países donde existen bosques de Polylepis.

\section{Materiales y Métodos}

\section{Área de estudio}

En Argentina, el género Polylepis encuentra su distribución más austral, desde el límite con Bolivia $\left(21^{\circ} 47^{\prime} \mathrm{S} ; 66^{\circ} 12^{\prime} \mathrm{O}\right)$ hacia el sur, incluyendo la Puna y zonas Altoandinas de Jujuy, las montañas más orientales de Salta y Jujuy, el cordón del Aconquija en Tucumán y Catamarca, y las Sierras de Córdoba y San Luis hasta los $32^{\circ} 43^{\prime}$ S-64 $52^{\prime}$ O (Cantero \& Bianco 1987; Braun Wilke et al. 2005). Todos son cordones montañosos con dirección N-S. En general, presentan una complejidad estructural considerable y en el Noroeste incluyen alturas que superan los 5000 m.s.n.m. Toda la distribución del género en
Argentina comparte un clima de montaña con heladas frecuentes durante el invierno, variaciones térmicas diarias considerables y una estacionalidad importante en las precipitaciones, con una estación seca marcada en el invierno.

\section{Modelos de distribución de especies}

Para la construcción de modelos predictivos de la distribución de los bosques de Polylepis en Argentina utilizamos datos de presencia, obtenidos en viajes al campo, bibliografía especializada (Simpson 1979; Cingolani et al. 2004), revisión y consulta de colecciones de herbarios de las Universidades Nacionales de Jujuy (JUA), Salta (MCNS), Instituto Miguel Lillo (LIL), Herbario Nacional de Bolivia (LPB) y Herbario San Marcos (USM, Peru), y consulta a las bases de datos disponibles en Internet [i.e, Trópico (www.tropicos.org) y GBIF (www.gbif.org)]. Trabajamos con 116 sitios de presencia para la especie P. tarapacana, 206 para P. tomentella, 348 para $P$. australis, 38 para $P$. crista-galli y 104 para $P$. hieronymi (Figura 1). Utilizamos menos datos de presencia de $P$. crista-galli debido a que la especie es poco abundante y fue descripta recientemente para Argentina. Las variables climáticas se obtuvieron del sitio web WorldClim (www.worldclim.org), las variables topográficas (altitud y pendiente) del sitio biogeo.ucdavis.edu, y los índices de aridez, evapotranspiración potencial anual y mensual de www.cgiar-csi.org (Trabucco \& Zomer 2009). Todas las variables utilizadas y los modelos de distribución resultantes, tienen una resolución de $0.74 \mathrm{~km}^{2}$. Se omitieron variables redundantes seleccionando aquellas que tuvieran una correlación de Pearson $<0.7$ y un mayor significado biológico de acuerdo a la experiencia de los autores.

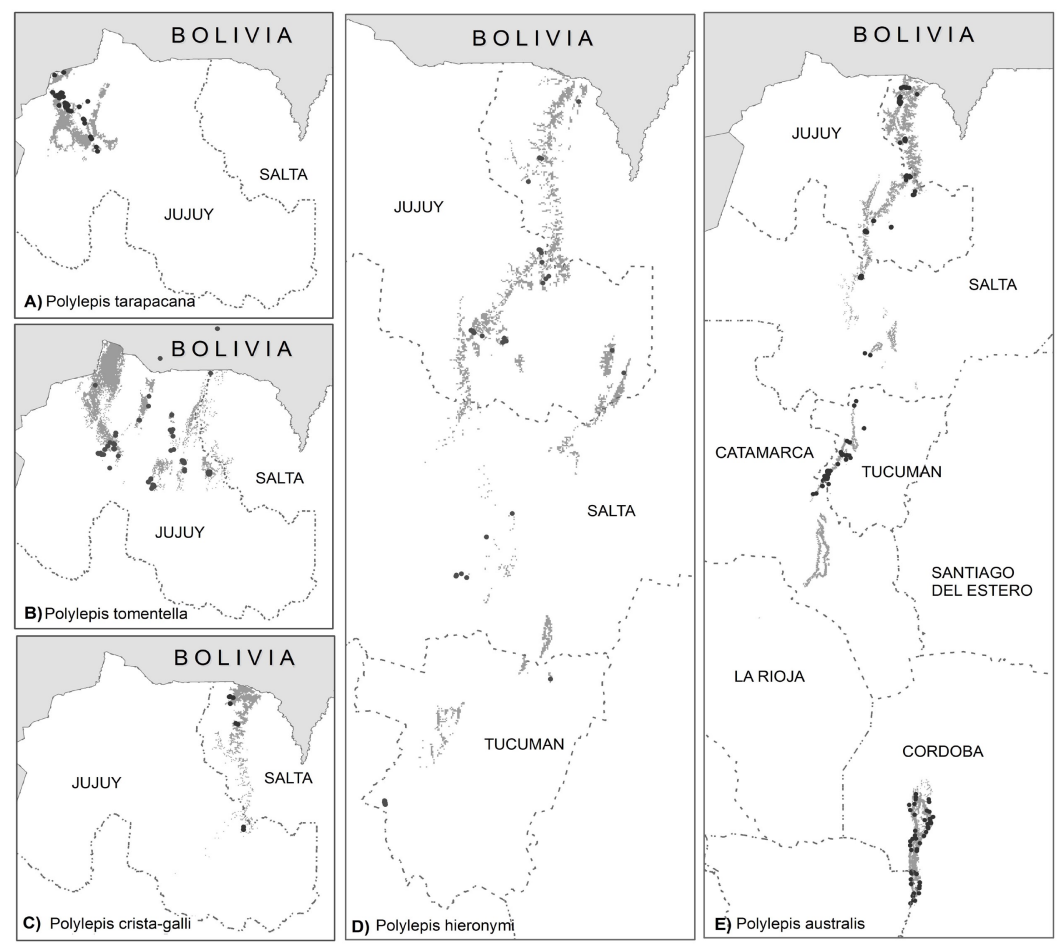

Figura 1. Distribución potencial de las especies de Polylepis descriptas en Argentina (en gris dentro de Argentina). Los círculos negros representan los puntos de presencia conocidos con los cuales se confeccionaron los modelos de distribución potencial.

Figure 1. Potential distribution of the Polylepis species described in Argentina (in gray within Argentina). Known presences with which the models were constructed are represented as black circles 
Para confeccionar mapas de las áreas de distribución potencial de las cinco especies de Polylepis en Argentina realizamos modelos predictivos de distribución de especies con el programa MaxEnt (Phillips et al. 2006). Utilizamos el promedio de 100 simulaciones, realizadas con los parámetros configurados por defecto en el programa. En cada una se utilizó al azar el 70\% de los datos de presencia para la construcción del modelo y el 30\% para la validación interna del mismo. La eficiencia general del modelo se estimó mediante el área bajo la curva de la característica operada por el receptor. El área utilizada para los modelos corresponde con las provincias del noroeste y centro de Argentina, de modo de cubrir la distribución de la especie con rango latitudinal más extenso ( $P$. australis). Por último, seleccionamos umbrales de corte para elaborar clasificaciones binarias de presencia-ausencia para cada especie en base al conocimiento de los autores: 0.7 para $P$. tarapacana, $P$. tomentella, $P$. crista-galli y $P$. hieronymi y 0.5 para $P$. australis.

\section{Amenazas, estado de conservación y áreas de referencia}

Entre los años 2006 y 2012 realizamos evaluaciones rápidas de los bosques en 490 parcelas de 60×60 m. Estas evaluaciones incluyeron al menos un ejemplar de Polylepis adulto. Las parcelas se distribuyeron en 43 localidades (entre 1 y 38 parcelas por localidad según el tamaño y la heterogeneidad del área y los bosques) e incluyeron áreas protegidas ( $N=6$ localidades) y no protegidas $(\mathrm{N}=37)$. De las 43 localidades visitadas, 8 correspondieron a $P$. tarapacana, 8 a $P$. tomentella, 5 a $P$. hieronymi, 1 a $P$. crista-galli y 21 a $P$. australis. Con el propósito de tener una mayor independencia de datos, consideramos una distancia mínima de $100 \mathrm{~m}$ entre parcelas y de $5 \mathrm{~km}$ entre localidades. En cada parcela evaluamos: a) presencia e identidad de especies de Polylepis, b) evidencias de tala, fuego, agricultura, ramoneo y especies leñosas exóticas, c) estado de conservación de los suelos de la parcela usando como indicador el porcentaje el suelo cubierto con vegetación [e.g., bosque, arbustales, pasturas (adaptado de Cingolani et al. 2003)], d) estado de conservación de la porción cubierta por bosque, utilizando un código del 1 al 5 , en el que se tuvo en cuenta la cobertura arbórea y la estructura del bosque (adaptado de Renison et al. 2011). La categoría 1 representó una cobertura arbórea $<30 \%$ y sin registros de renovales (definidos como individuos entre 5 y $100 \mathrm{~cm}$ de altura). La categoría 2 tuvo una cobertura arbórea $<30 \%$, pero con presencia de renovales. La categoría 3 presentó una cobertura $>30 \%$ y dosel arbóreo a $<2 \mathrm{~m}$ de altura. La categoría 4 mostró una cobertura $>30 \%$ y dosel arbóreo entre 2 y $5 \mathrm{~m}$ de altura. La categoría 5 presentó una cobertura $>30 \%$ y el dosel arbóreo $>5 \mathrm{~m}$ de altura. Dado el carácter exploratorio de las evaluaciones no realizamos estadísticas formales, sólo presentamos valores medios por especie, su rango de variabilidad y describimos en mayor detalle las localidades mejor conservadas según nuestros indicadores con el fin de tenerlos de forma provisoria como sitios de referencia.

\section{Resultados}

\section{Modelos de distribución}

La distribución potencial a escala regional de cada especie se puede observar en la Figura 1. La eficiencia general de los modelos de distribución, evaluada con el valor de AUC, estuvo por encima de 0.98 en todos los casos $(P$. tarapacana 0.993, P. tomentella 0.982, P. australis 0.993, P. crista-galli 0.993, P. hieronymi 0.991).

En ambientes altoandinos y de puna encontramos a $P$. tarapacana y $P$. tomentella. Las poblaciones de $P$. tarapacana estuvieron limitadas a ambientes altoandinos del noroeste de la provincia de Jujuy. Los modelos sugirieron su presencia entre 4000 y 5000 m.s.n.m, con una superficie cubierta de $1650 \mathrm{~km}^{2}$ (Figura 1A). Polylepis tomentella se distribuyó en los ambientes de puna, a menor altitud y más al este que $P$. tarapacana. Los modelos predijeron su posible presencia entre 2700 y 4600 m.s.n.m., con una superficie cubierta de $3400 \mathrm{~km}^{2}$ (Figura 1B).

En ambientes montanos húmedos encontramos a P. australis, P. crista-galli y P. hieronymi. Para P. australis (Figura 1E) los modelos sugirieron un rango altitudinal entre 900 y 4000 m.s.n.m y una distribución latitudinal desde el extremo sur de Bolivia hasta el centro sur de la provincia de Córdoba, con una extensión de $1200 \mathrm{~km}$. La distribución modelada incluyó áreas de pastizales de altura y el ecotono con el bosque montano de yungas en Jujuy, Salta y Tucumán, y con el chaco serrano en Córdoba y San Luis. Los modelos estimaron un área potencial para $P$. australis de $7600 \mathrm{~km}^{2}$. En el caso de P. cristagalli los modelos sugirieron una distribución potencial que se superpuso ampliamente con la de $P$. australis y parcialmente con la de $P$. hieronymi, en un rango altitudinal entre 1800 y 3800 m.s.n.m, con una superficie de 1050 km² (Figura 1C). Los modelos para P. hieronymi (Figura 1D) sugirieron un rango altitudinal entre 1500 y 2900 m.s.n.m, superponiéndose con $P$. australis en las provincias de Salta y Jujuy, donde $P$. hieronymi se extendió hacia zonas más bajas y orientales y se mezcló con el bosque montano de yungas. Otra diferencia con $P$. australis es el gradiente latitudinal que ocupó, ya que $P$. hieronymi no llegó a la provincia de Córdoba y cubrió una superficie de $2600 \mathrm{~km}^{2}$. 
Amenazas, estado de conservación y áreas de referencia

Si tomamos en cuenta el promedio ponderado por localidad, encontramos evidencias de fuego en $80 \%$ de las parcelas para $P$. tarapacana y $P$. tomentella, $60 \%$ para $P$. australis, $0 \%$ en el caso de $P$. crista-galli y $40 \%$ para $P$. hieronymi. Las evidencias de tala fueron menores a las de fuego, con evidencias de tala en $30 \%$ de las parcelas situadas en bosques de $P$. tarapacana, $29 \%$ en $P$. tomentella, $26 \%$ en $P$. australis (principalmente en Salta y Jujuy), $0 \%$ en $P$. crista-galli y $20 \%$ en $P$. hieronymi. Registramos evidencias de ramoneo en casi todas las localidades, aunque pudimos observar que ante la misma accesibilidad al ganado, las especies $P$. tarapacana y $P$. tomentella se encontraban bastante menos ramoneadas que las especies $P$. australis y $P$. hieronymi. Los grandes herbívoros registrados en la mayoría de las localidades visitadas fueron bovinos, equinos y ovinos. En la región noroeste de Argentina registramos camélidos sudamericanos (llamas, vicuñas y guanacos) y asnales (en parte asilvestrados). Registramos porcinos en la provincia de Córdoba, con remoción de suelo debido a sus hábitos alimentarios, y ciervos exóticos en la provincia de Tucumán. No detectamos signos de agricultura en ninguna de las parcelas.

Según nuestros registros, la única especies de Polylepis afectada por invasiones por especies leñosas exóticas es $P$. australis. Fuera de las parcelas registramos plantaciones de Pinus sp. en extensas áreas en la provincia de Córdoba, y puntualmente en el parque provincial Lagunas de Yala en Jujuy. Algunas de estas plantaciones fueron implantadas sobre bosque de Polylepis, otras en sitios muy cercanos. Registramos pinos escapados de las plantaciones en 2 parcelas. En la provincia de Córdoba también observamos otras especies exóticas invadiendo los bosques de $P$. australis, incluyendo las leñosas Cotoneaster franchetii, Betula pendula, Rosa rubiginosa, Rubus ulmifolius, Salix viminalis y $S$. aff fragilis ( $\mathrm{N}=5$ parcelas).
Los indicadores del estado de conservación registrados por especie se resumen en la Tabla 1. Los bosques evaluados de $P$. tarapacana $(\mathrm{N}=8$ localidades) tenían una cobertura y estructura relativamente rala y simple. El Volcán Granada (provincia de Jujuy, 22 $2^{\circ} 1^{\prime} 43.6^{\prime \prime} \mathrm{S}$ - 66 36'10.6" O, entre 4300 y 4492 m.s.n.m.) fue la localidad con los bosques mejor conservados. En el Volcán Granada ( $\mathrm{N}=7$ parcelas), las coberturas de bosque alcanzaron $30 \%$ por parcela, el índice de estructura varió entre 2 y 3 , encontramos individuos de hasta $3 \mathrm{~m}$ de alto y el suelo presentó un promedio de cobertura vegetal de $79.2 \%$ (varió entre el 60 y el $80 \%$ ).

Los bosques evaluados de la especie $P$. tomentella ( $\mathrm{N}=8$ localidades) fueron variables en su estructura (Tabla 1). Los bosques mejor conservados fueron los cercanos a la localidad de Árbol Solo (provincia de Jujuy, 22 $2^{\circ} 5^{\prime} 32.5^{\prime \prime}$ S - 655' $12.4^{\prime \prime} \mathrm{O}$, entre 3980 y 4083 m.s.n.m.). En Árbol Solo ( $\mathrm{N}=6$ parcelas) la cobertura de bosque fue de hasta el 55\% de la parcela, su índice de estructura varió entre 2 y 5 , presentó individuos de hasta $8 \mathrm{~m}$ de alto y suelos con coberturas de vegetación promedio de $83.7 \%$ (varió entre el 70 y el 95\%).

Los bosques de la especie $P$. australis $(\mathrm{N}=21$ localidades) fueron los que mayor variabilidad presentaron en cuanto a su estado de conservación, con bosques muy ralos y cobertura promedio de suelos tan baja como $31.6 \%$. P. australis también fue la especie con los bosques más extensos registrados en este trabajo, los cuales se encuentran en el sector sur del Parque Nacional Quebrada del Condorito y campos privados circundantes $\left(10 \mathrm{~km}^{2}, 31^{\circ} 45^{\prime} 30.1^{\prime \prime} \mathrm{S}-64^{\circ} 46^{\prime} 01.2^{\prime \prime} \mathrm{O}\right)$, en la provincia de Córdoba. Los bosques mejor conservados fueron los bosques cercanos a la localidad de Los Molles, en la provincia de

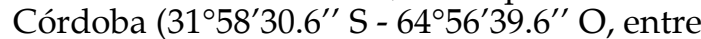
1500 y 2650 m.s.n.m.). Estos bosques fueron evaluados en 30 parcelas, tuvieron coberturas de hasta 95\% de la parcela, su estructura varió

Tabla 1. Promedios \pm error estándar y (rango) para dos indicadores del estado de conservación de 490 parcelas con bosques de Polylepis distribuidas en 43 localidades de Argentina: 1) proporción (\%) del suelo cubierto con vegetación, y 2) cobertura y estructura del bosque codificados de 1 a 5 , donde 1 fueron los bosques menos densos y estructuralmente más simples y 5 los más densos y complejos (ver Materiales y Métodos). En el encabezado de las columnas se detalla la especie de Polylepis y el número de localidades sobre los que están basados los promedios.

Table 1. Averages \pm standard error and (range) for two indicators of conservation state in 490 plots with Polylepis forests distributed in 43 localities of Argentina (details in legend in Spanish).

\begin{tabular}{lccccc}
\hline & $\begin{array}{c}\text { P. tarapacana } \\
(\mathrm{N}=8)\end{array}$ & $\begin{array}{c}\text { P. tomentella } \\
(\mathrm{N}=8)\end{array}$ & $\begin{array}{c}\text { P. australis } \\
(\mathrm{N}=21)\end{array}$ & $\begin{array}{c}\text { P. crista-galli } \\
(\mathrm{N}=1)\end{array}$ & $\begin{array}{c}\text { P. hieronymi } \\
(\mathrm{N}=5)\end{array}$ \\
\hline Suelo cubierto con & $68.2 \pm 2.0$ & $78.5 \pm 1.6$ & $85.9 \pm 3.4$ & 90.0 & $97.7 \pm 1.0$ \\
vegetación (\%) & $(60.0$ a 79.2$)$ & $(70.0$ a 83.7$)$ & $(31.6$ a 100$)$ & - & $(95.0$ a 100$)$ \\
Índice de cobertura y & $1.9 \pm 0.2$ & $2.8 \pm 0.3$ & $2.2 \pm 0.2$ & 4.0 & $3.3 \pm 0.5$ \\
estructura del bosque & $(1.0$ a 2.5$)$ & $(2.0$ a 4.0$)$ & $(1.0$ a 3.7) & - & $(2.1$ a 5.0) \\
\hline
\end{tabular}


según la parcela entre 1 y 5, encontramos individuos de hasta $16 \mathrm{~m}$ de alto y coberturas de vegetación entre 80 y 100\% del suelo.

Los bosques de $P$. crista-galli fueron evaluados en una única localidad (cercana a Alto Calilegua, 233ㄴ $44.4^{\prime \prime} \mathrm{S}-64^{\circ} 53^{\prime} 56.8^{\prime \prime}$ O) mediante 2 parcelas de características similares. Para esta especie encontramos coberturas de bosque de $35 \%$, estructura con índice de 4 y suelos cubiertos con vegetación en un $90 \%$.

Los arbustos y árboles de P. hieronymi $(\mathrm{N}=5$ localidades) generalmente aparecieron como individuos aislados o en parches de hasta 1200 $\mathrm{m}^{2}$, inmersos en bosques dominados por otras especies típicas de las yungas altas. Esto los diferencia de las otras especies de Polylepis estudiadas, que suelen dominar la canopia de los arbustales y bosques que conforman. La localidad evaluada con poblaciones de $P$. hieronymi mejor conservados fue en las cercanías de Tiraxi (provincia de Jujuy, $31^{\circ} 58^{\prime} 30.6^{\prime \prime} \mathrm{S}-64^{\circ} 56^{\prime} 39.6^{\prime \prime} \mathrm{O}$, entre $1848 \mathrm{y}$ 2134 m.s.n.m.). Aquí evaluamos 6 parcelas con coberturas de $P$. hieronymi de hasta $35 \%$ por parcela, siempre en parches menores a $600 \mathrm{~m}^{2}$. El índice de estructura de estos bosques varió entre 3 y 5, excepcionalmente encontramos individuos de hasta $8 \mathrm{~m}$ de alto y cobertura vegetal del suelo entre 75 y 100\%.

\section{DiscUSIÓN}

\section{Modelos de distribución de especies}

El área de distribución predicha por los modelos corresponde, en el mejor de los casos, al ambiente que potencialmente podrían ocupar las especies. El área realmente ocupada puede ser menor, y depender de factores que nuestros modelos no incluyeron, como las consideraciones biogeográficas y las interacciones con el uso humano (Fjeldså \& Kessler 2004). En algunos casos, los modelos desarrollados predijeron áreas de presencia para algunas especies en sitios alejados de la distribución conocida, como la presencia de $P$. hieronymi en la provincia de Tucumán y de $P$. tarapacana, a más de $100 \mathrm{~km}$ al sur del límite con Bolivia y en alturas de hasta 5000 m.s.n.m. Estos sitios fueron confirmados a campo durante verificaciones posteriores, lo que demostró la utilidad de los modelos. También señalan a $P$. australis en la provincia de Catamarca más al sur de su distribución conocida y en una porción en Bolivia cerca del límite con Argentina (no reportado en la Figura 1). Aún queda por confirmar si la especie se encuentra en esos sitios. Los mapas -además- indican la presencia de $P$. australis en la Quebrada de Humahuaca (provincia de Jujuy, protuberancia hacia el oeste-norte) (Figura 1E), donde no hay registros actuales de la especie. Dada la accesibilidad que presenta la Quebrada de Humahuaca para las observaciones botánicas, es poco probable que la especie haya pasado inadvertida. Se podría hipotetizar, entonces, que esta región habría tenido a $P$. australis en el pasado, y que su ausencia en el presente se debería a la ocupación humana continua durante milenios. Otra posibilidad es que esta región sólo recientemente haya tenido condiciones favorables para el establecimiento y crecimiento de $P$. australis, pero que ésta aún no ha llegado.

\section{Amenazas, estado de conservación y áreas de referencia}

La incidencia de signos de fuegos registrada en este trabajo fue alta en casi todas las especies. Muchos de los fuegos son causados para producir rebrote como alimento para el ganado (para $P$. australis ver Renison et al. 2002, 2006) o para obtener leña seca (observaciones personales en bosques de $P$. tarapacana y $P$. tomentella). Todas las especies son ramoneadas y los registros de tala si bien menores a los de fuego también estuvieron presentes. Estas observaciones sugieren que los fuegos, la tala y el ramoneo son disturbios que inciden en los bosques de Polylepis en toda su extensión, y que una reducción de dichos disturbios aumentaría la superficie cubierta por estos bosques y su complejidad estructural (para P. australis Cingolani et al. 2008; Renison et al. 2011). Sin embargo, se necesitan más estudios para determinar si los niveles de disturbio observados son aceptables. También observamos invasiones incipientes de leñosas en bosques de Polylepis, hecho que debe ser atendido por las autoridades de conservación ya que una reciente revisión de Pauchard et al. (2009) señala cómo las actividades humanas crecientes en las montañas están fomentando las invasiones por especies exóticas de estos sistemas. En las Sierras de Córdoba, Giorgis et al. (2011) señalan como el principal factor que influye en la presencia de exóticas a la distancia a casas y caminos (y por ende a fuentes de propágulos).

En relación a los posibles efectos del cambio climático, Morales et al. (2004) encontraron en el noroeste Argentino que el crecimiento 
de $P$. tarapacana está controlado de manera significativa por las precipitaciones, las cuales han disminuido en las últimas décadas (Carilla et al. datos no publicados). Es posible que estos cambios afecten a los patrones de mortalidad, crecimiento y establecimiento de esta especie que ya de por si crece muy cercano a los límites fisiológicos impuestos por la altura. En Córdoba, los posibles efectos del calentamiento global sobre los bosques de $P$. australis son discutidos por Marcora et al. (2008). Consideraciones generales indican que el calentamiento global sería más preocupante en las montañas bajas, donde Polylepis no podrá desplazarse altitudinalmente a zonas más elevadas, y en los sitios con más ganadería, tala y fuegos, donde los bosques de Polylepis están confinados a micrositios protegidos y con menos posibilidades de migrar por la matriz ganadera para encontrar su óptimo en clima y escapar de posibles eventos climáticos extremos (Innes et al. 2009).

Las evaluaciones rápidas del estado de conservación de los bosques son útiles para la determinación de áreas de referencia. Estas áreas de referencia sirven para planear proyectos de conservación y restauración, establecer metas de conservación y evaluar el grado de éxito de las acciones realizadas sobre la base de la comparación entre las áreas a restaurar/conservar y las áreas de referencia (SER 2004; www.ser.org). Es importante para la conservación de la biodiversidad, de los bosques per se y de los bienes y servicios que producen, que existan rodales en todos los estados de desarrollo, desde bosques maduros hasta bosques recién disturbados y en proceso de regeneración (Lindenmayer et al. 2006; McComb 2008). Aquí sugerimos que las áreas mejor conservadas en nuestro estudio sean tomadas como referencia, sin dejar de reconocer todas las limitaciones que puedan tener y siempre atentos a mejores opciones. De esta forma, y a modo de ejemplo, se podría argumentar que las áreas protegidas que incluyen a $P$. australis deberían apuntar a tener un mosaico de parches que incluyen sitios con más de $30 \%$ de cobertura de bosque y un dosel arbóreo $>5 \mathrm{~m}$ de altura, y así sucesivamente con cada una de las variables descriptas para la localidad de referencia propuesta.

Dado que evaluamos el estado de conservación en seis localidades situadas en áreas protegidas (todas en bosques de $P$. australis), es sorprendente que estas localidades no fueron las mejor conservadas y las sugeridas como las de referencia. Sin embargo, las únicos dos Parques Nacionales que incluyen una porción importante con bosques de Polylepis (Campo de los Alisos y Quebrada del Condorito) tenían menos de 12 años desde su creación cuando evaluamos el estado de conservación. Este tiempo no es suficiente para recuperar la cobertura y estructura de estos bosques de montaña debido a sus tasas de crecimiento reducidas. Evaluamos parcelas en el Parque Provincial Lagunas de Yala (Provinica de Jujuy, N=25) que según nuestros registros resultaron menos conservados que el promedio de las parcelas, y en la Reserva Hídrica Pampa de Achala (Provincia de Córdoba, 3 localidades distribuidas en 57 parcelas) donde describimos la localidad de referencia para $P$. australis. La Reserva Hídrica Pampa de Achala también era de reciente formación, y la localidad de referencia seguramente debe su estado de conservación a la gran distancia a asentamientos humanos y pendientes pronunciadas que dificultan el acceso del ganado en la zona (Renison et al. 2010, 2011).

En los bosques de P. australis de Córdoba, la cobertura vegetal del suelo es un buen indicador de la erosión de suelos dado que en estos escarpados ambientes es muy difícil que el suelo se conserve al desaparecer la cobertura vegetal (Cingolani et al. 2008; Renison et al. 2010). En este contexto, preocupan las bajas coberturas vegetales que registramos en muchas de las parcelas evaluadas. Además, en parcelas con baja cobertura vegetal era muy frecuente observar raíces descubiertas, piedras sostenidas por un pilar de suelo y otras evidencias de pérdida de suelo. Consideramos que los porcentajes mínimos de cobertura vegetal (Tabla 1) de muchas localidades con $P$. australis son excesivamente bajos (e.g., 31.6\%). En cambio, para ambientes alto andinos y de puna ( $P$. tarapacana y $P$. tomentella) no hay un consenso sobre si la falta de cobertura vegetal necesariamente desencadena pérdida neta de suelos, tema que merece más estudios (e.g., Guzmán 1997). A modo de ejemplo, Preston (2003) sugiere que en el Altiplano Boliviano de la zona de Tarija la pérdida y deposición de suelo están en equilibrio, mientras que Fjeldså \& Kessler (2004) hacen una síntesis donde sugieren que los fuegos y la carga ganadera excesiva han desencadenado procesos de erosión generalizados. De acuerdo a nuestro conocimiento no existen bosques de Polylepis en la zona alto andina y de puna en áreas sin ganadería y sin quema de pasturas, por lo cual será muy difícil resolver estas controversias sobre el efecto de la ganadería y fuego en el corto plazo. 


\section{Estrategias de conservación}

A favor de la conservación de los bosques de Polylepis, se ha registrado disminución de la ganadería a menos de la mitad en los últimos 50 años en la vertiente oriental de las montañas del noroeste argentino, donde se distribuyen $P$. crista galli, $P$. hieronymi y $P$. australis (Aráoz \& Grau 2010). También se ha registrado disminución de la ganadería y de la población rural en los departamentos de la Puna jujeña, donde se distribuyen $P$. tomentella y P. tarapacana (Izquierdo \& Grau 2008). Por otra parte, las poblaciones de vicuñas han aumentado de forma significativa, con efectos desconocidos pero posiblemente negativos para $P$. tarapacana. Por ello, es de suponer que si las poblaciones de las distintas especies de Polylepis han soportado las altas presiones ganaderas del pasado, con menos ganado las poblaciones pueden mantenerse estables o incluso se podrán recuperar lentamente, hecho que amerita estudios para verificarlo.

En muchas de las regiones visitadas sería importante aumentar la cobertura vegetal de los suelos ya que, al menos en el centro Argentino, la erosión de los suelos está muy relacionada a la baja cobertura vegetal provocada por ganadería y fuegos (Cingolani et al. 2004; Renison et al. 2010). Asimismo, la alta incidencia de fuego encontrada en casi todos los bosques visitados, sugiere que disminuir la quema sería una herramienta muy importante para lograr una recuperación de los bosques. Además, al menos P. australis es muy ramoneado por el ganado doméstico, y en el área de distribución de esta especie sería importante disminuir las cargas de ganado (Giorgis et al. 2010). La inaccesibilidad de los actuales bosques de Polylepis juega un papel importante en su conservación. En las provincias de Jujuy, Salta, Córdoba y San Luis se ha observado la construcción y/o mejora de caminos en cuyo trayecto hay bosques de Polylepis que deberían tener asegurado su amparo, ya que en otras regiones se ha constatado que la presencia de caminos facilita la degradación y desaparición de bosques, incluyendo los de Polylepis (Purcell \& Brisford 2004; Cingolani et al. 2008).

¿Cómo lograr los objetivos de conservación? Esta es una pregunta que cada región deberá resolver de acuerdo con las idiosincrasias, ambientes y posibilidades locales. Entre las alternativas se pueden mencionar la búsqueda de manejos ganaderos más compatibles con la conservación de estos bosques, la delimitación de árboles o zonas semilleras intangibles para promover la regeneración de bosques, la generación de incentivos económicos para la conservación de bosques, y la estimulación de la valoración ética y estética de estos ambientes. Por otro lado, es necesario incluir a las comunidades locales que hacen uso de la leña de Polylepis, ya que ellos necesitan de esa fuente energética y por ende deberían tener interés en conservarla.

La creación de nuevas áreas protegidas mediante expropiación de las propiedades y la contratación del personal que en ellas habita para cuidar el área protegida y la consolidación deáreas protegidas existentes seguramente son las estrategias de conservación más efectivas. En Argentina existen 23 áreas protegidas que abarcan sitios con potencialidad para algunas de las especies de Polylepis (Figura 1) (Chebez 2005; Información Suplementaria, Apéndice 2). A pesar de las numerosas áreas protegidas donde se encuentran bosques, la mayoría son deficientes en su efectividad de protección, una realidad compartida con muchos países (Soulé \& Wilcox 1980; Wright et al. 2007). La representación de las especies de Polylepis dentro de áreas efectivamente protegidas es nula en el caso de $P$. tarapacana, y escasa para $P$. tomentella, $P$. crista-galli y $P$. hieronymi. Solamente $P$. australis se encuentra dentro de varias áreas protegidas que incluyen dos Parques Nacionales, pero incluso esta especie tiene menos superficie protegida que lo aconsejado para ambientes de montaña (Hamilton 2002). En sitios donde la recuperación natural de los bosques es poco probable, es importante fomentar iniciativas de reforestación con especies nativas, incluyendo Polylepis. En la provincia de Córdoba ya existen al menos tres grupos que han forestado de forma exitosa con miles de plantines de $P$. australis (Renison et al. 2005; www.ecosiste masarg.cor.ar; www.reforestacion.com.ar y www.fundacionab.org/restauracionecologica).

\section{Futuros estudios}

Consideramos necesario realizar estudios en las siguientes líneas: 1) profundizar sobre la ecología básica de todas las especies del género como se ha hecho para $P$. australis. Si bien las especies de Polylepis pueden ser similares morfológicamente, los últimos estudios muestran que muchos aspectos de sus estrategias de vida pueden diferir entre especies y en respuesta al ambiente, 2) modelar la dinámica de los bosques de $P$. australis (donde ya hay suficientes estudios) con el fin de investigar distintos escenarios de 
manejos ganaderos, fuegos, talas, invasiones con especies exóticas y de cambio climático (e.g., Favier et al. 2004), 3) determinar tasas de pérdida de suelos en bosques de Polylepis, sus causas y cómo reducirlas (e.g., Chartier et al. 2009; Landi \& Renison 2010), 4) profundizar el estudio sobre los efectos del fuego en la dinámica de los bosques y sus suelos (e.g., Vivian et al. 2008), 5) determinar las tendencias en la migración humana, extracción de leña, uso ganadero de las zonas montañosas que nos ayudarán a predecir las futuras posibilidades de conservación y amenazas a los bosques (e.g., Grau \& Aide 2007), 6) mejorar las evaluaciones de la biota asociada a los bosques y determinar sus necesidades de conservación (e.g., Robledo \& Renison 2010; Bellis et al. 2009; Verga et al. 2012), 7) desarrollar técnicas de restauración de bosques con especies nativas para evitar el uso de especies exóticas que pueden ocasionar problemas (e.g., Picchetti et al. 2004; Renison et al. 2005; South et al. 2005), 8) determinar los métodos más económicos y factibles para controlar la invasión de especies no nativas (Kettenring et al. 2011), 9) desarrollar estudios palinológicos para determinar lluvia polínica del pasado, relacionar con el clima pasado y escenarios futuros como los realizados por Lupo et al. (2006) en el noroeste argentino y Gosling et al. (2009) en Bolivia, 10) estudiar cómo influyen los bosques de Polylepis en el rendimiento hídrico, ya que frecuentemente se los menciona como importantes para el suministro de agua (e.g., Fjeldså \& Kessler 2004) pero no existen estudios que lo avalen, 11) estudiar el efecto del aumento de poblaciones de herbívoros nativos sobre la dinámica de regeneración, principalmente de vicuñas en relación con Polylepis tarapacana y reintroducción de guanacos en el PN Condorito en relación con Polylepis australis.

En Argentina existen extensas áreas de bosques de Polylepis, en su mayoría muy degradados por ganadería, fuegos y tala, pero que constituyen una excelente plataforma para su recuperación y expansión y para promover la formación de más bosques maduros con todo lo que ello implica en términos de biodiversidad y funcionamiento del ecosistema. Asimismo, existe un incremento en las actividades de conservación relacionadas a los bosques de Polylepis y en el número de áreas protegidas. El conocimiento sobre los bosques de Polylepis aún es escaso pero existen tendencias de incremento muy alentadoras y que deben ser promovidas.

Agradecimientos:Agradecemos a American Bird Conservancy por la financiación. Los pobladores de las regiones que visitamos por las ayudas y permisos. Los colegas A. Grau, R. Lobo, L. Malizia, R. Neumann, L.J. Novara, R.I. Meneses y W. Mendoza proveyeron datos de presencia o nos orientaron hacia ellos. U. Colina, R. Cáceres, H. Mollo, M. Reader, M. Burgos, G. Cruz, K. Schittek, D. Schinner y G. Torres nos ayudaron en el campo. R. Braun Wilke, R. Suárez, C. Coutsiers y dos revisores anónimos mejoraron el trabajo con sus sugerencias. A las autoridades del Parques Nacionales Quebrada del Condorito, Campo Los Alisos y Parque Provincial Laguna de Yala por los permisos.

\section{BiBLIOGRAFÍA}

Aráoz, E \& HR Grau. 2010. Fire mediated forest encroachment in response to climatic and land use change in subtropical Andean treelines. Ecosys., 13:992-1005.

Bellis, LM; L Rivera; N Politi; E Martín; ML Perasso; et AL. 2009. Latitudinal patterns of bird richness, diversity and abundance in Polylepis australis mountain forest of Argentina. Bird Cons. Int., 19:265-276.

Braun Wilke, RH; LPE Picchetti \& GF Guzmán. 2005. Desaparición de Queñoales de la Puna Jujeña (NOArgentino). Agraria, 2:73-77.

CANTERO, JJ \& CA Bianco. 1987. El límite austral de Polylepis australis Bitt. (Tabaquillo) en la República Argentina. Parodiana, 5:65-71.

Chartier, MP; CM Rostagno \& FA Roig. 2009. Soil erosion rates in rangelands of northeastern Patagonia: A dendrogeomorphological analysis using exposed shrub roots. Geomorphology, 106:344-351.

Chebez, JC. 2005. Guía de las Reservas Naturales de la Argentina. Tomo 4 y 5. Editorial Albatros, Buenos Aires, Argentina.

Cierjacks, A; S Salgado; K Wesche \& I Hensen. 2008. Post-fire population dynamics of two tree species in high-altitude Polylepis forests of Central Ecuador. Biotropica, 40:176-182.

Cingolani, AM; D Renison; MR ZAK \& MR CABIDO. 2004. Mapping vegetation in a heterogeneous mountain rangeland using Landsat data: an alternative method to define and classify land-cover units. Remote Sens., 92:84-97.

Cingolani, AM; D Renison; P Tecco; D Gurvich \& M CABIDO. 2008. Predicting cover types in a mountain range with long evolutionary grazing history: a GIS approach. J. Biogeogr., 35:538-551.

Favier, C; J Chave; A Fabing; D Schwartz \& MA Dubois. 2004. Modelling forest-savanna mosaic dynamics in man-influenced environments: effects of fire, climate and soil heterogeneity. Ecol. Model., 171:85-102.

FJELDSÅ, J \& M KESSLER. 2004. Conservación de la biodiversidad de los bosques de Polylepis de las tierras Altas de Bolivia. Una contribución al manejo sustentable en los Andes. DIVA Dinamarca. Pp 214.

Giorgis, M; AM Cingolani; I Teich; D Renison \& I Hensen. 2010. Do Polylepis australis trees tolerate herbivory? Seasonal patterns of shoot growth and its consumption by livestock. Plant Ecol., 207:307-319.

Giorgis, M; P Tecco; AM Cingolani; D Renison; P Marcora; ET AL. 2011. Factors associated with woody alien species distribution in a newly invaded mountain system of central Argentina. Biol. Invas., 13:1423-1434.

Gosling, WD; JA Hanselman; C Knox; BG Valencia \& MB Bush. 2009. Long-term drivers of change in Polylepis woodland distribution in the central Andes. J. Veg. Sci., 20:1041-1052.

GraU, HR \& TM Aide. 2007. Are rural-urban migration and sustainable development compatible in mountain 
systems? Mount. Res. and Develop., 27:119-123.

GuZMÁN, GF. 1997. In uencia del pastoreo en la regeneración del bosque de queñoa (Polylepis tomentella Wedd.) en la puna de Jujuy (Argentina). Tesis de Maestría en Conservación y Gestión del Medio Natural. Universidad Internacional de Andalucía-España.

HamiLton, LS. 2002. Conserving mountain biodiversity in protected areas. Pp. 295-306 en: Körner, C y EM Spehn (eds.). Mountain Biodiversity: A global assessment. Parthenon, New York, U.S.A.

InNeS, J; LA Joyce; S KellomäKI; B Louman; A Ogden; et AL. 2009 Management for adaptation. Pp. 135-169 en: Seppälä R, A Buck \& P Katila (eds.). Adaptation of forests and people to climate change - A global assessment report. Esa - Print Oy, Finlandia.

IzQuiERDo, AE \& HR Grau. 2009. Agriculture adjustment, land-use transition and protected areas in Northwestern Argentina. J. Environ. Manage., 90:858-65.

Kessler, M. 2002. The "Polylepis problem": where do we stand? Ecotropica, 8:97-110.

Kessler, M \& AN Schmidt-Lebuhn. 2006. Taxonomical and distributional notes on Polylepis (Rosaceae). Org. Diver. Evol., 5:1-10.

KetTENRING, KM \& CR AdAMs. 2011. Lessons learned from invasive plant control experiments: a systematic review and meta-analysis. J. Appl. Ecol., 48:970-979.

Landi, M \& D Renison. 2010. Forestación con Polylepis australis en suelos erosionados de las Sierras Grandes de Córdoba: evaluación del uso de terrazas y vegetación nodriza. Ecol. Aust., 20:47-55.

LindenMayer, D; J FrankLin \& J Fischer. 2006. General management principles and a checklist of strategies to guide forest biodiversity conservation. Biol. Conserv., 131:433-445.

Lupo, LC; MM Bianchi; E Aráoz; R Grau; C Lucas; et AL. 2006. Climate and human impact during the past 2000 years as recorded in the Lagunas de Yala, Jujuy, northwestern Argentina. Quarter. Intern., 158:30-43.

Marcora, P; J Hensen; D Renison; P Seltmann \& K Wesche. 2008. The performance of Polylepis australis trees along their entire altitudinal range: implications of climate change for their conservation. Diver. Distrib., 14:630-636.

МсСомв, ВС. 2008. Wildlife habitat management: concepts and applications in forestry. CRC Press. New York, USA.

Morales, MS; R Villalba; HR Grau \& L Paolini. 2004. Rainfall-controlled tree growth in high-elevation subtropical treelines. Ecology, 85:3080-3089.

NAVArro, G;S Arrázola;JA ValderRAma;W Ferreira;N De La BARRA; ET AL. 2010. Diagnóstico del estado de conservación y caracterización de los bosques de Polylepis en Bolivia y su avifauna. Rev. Bol. Ecol. y Cons. Amb., 28:1-35.

Paduano, GM; MB Bush; PA Baker; SC Fritz \& GO Seltzer. 2003. A vegetation and fire history of Lake Titicaca since the Last Glacial Maximum. Palaeogeog. Palaeoclimat. Palaeoecol., 194:259-279.

Pauchard, A; C Kueffer; H Dietz; CC Daehler; J AleXANDER; Et Al. (2009) Ain't no mountain high enough: plant invasions reaching new elevations. Front. Ecol. Environ., 7:479-486.

Phillips, SJ; RP ANDERson \& RE Schapire. 2006. Maximum entropy modeling of species geographic distributions. Ecol. Model., 190:231-259.

Picchetti, LP; BS Villafañe \& GF GuZmán. 2004. Ensayos de germinación en Polylepis spp. (Rosaceae), "queñoa", de rodales de la puna jujeña. Agraria, 1:71-75.

Preston, D; J Fairbairn; N Paniagua; G MaAs; M YeVara; et AL. 2003. Grazing and environmental change on the Tarija Alitplano, Bolivia. Mount. Res. Devel., 23:141-148.

PURCELL, J \& A BRISFORD. 2004. Reassessing the causes of decline of Polylepis, a tropical subalpine forest. Ecotropica, 10:155-158.

Renison, D; AM Cingolani \& R Suárez. 2002. Efectos del fuego sobre un bosquecillo de Polylepis australis (Rosaceae) en las montañas de Córdoba, Argentina. Rev. Chil. Hist. Nat., 75:719-727.

RenISON, D; AM CingOlani; R SuÁreZ; E MENOYO; C COUTSIERS; ET AL. 2005. The restoration of degraded mountain forests: effects of seed provenance and microsite characteristics on Polylepis australis seedling survival and growth in Central Argentina. Rest. Ecol., 13:129-137.

Renison, D; I Hensen; R Suárez \& AM Cingolani. 2006. Cover and growth habit of Polylepis woodlands and shrublands in the mountains of central Argentina: human or environmental influence? J. Biogeog., 33:876-887.

Renison, D; I Hensen; R SuÁreZ; AM Cingolani; P Marcora; ET AL. 2010. Soil conservation in Polylepis mountain forests of Central Argentina: Is livestock reducing our Natural capital? Austral Ecol., 35:435-443.

Renison, D; I Hensen \& R SuÁrez. 2011. Landscape structural complexity of high-mountain Polylepis australis forests: A new aspect of restoration goals. Restor. Ecol., 19:390-398.

RoBLEDO, GL \& D RENISON. 2010. Wood-decaying polypores in the mountains of central Argentina in relation to Polylepis forest structure and altitude. Fungal Ecol., 3:178-184.

SER 2004. Mission \& Vision of the Society for Ecological Restoration Internacional. Society for Ecological Restoration (www.ser.org, consultado 03/10/2012).

SIMPSON, BB. 1979. A revision of the Genus Polylepis (Rosaceae: Sanguisorbeae). Smithsonian Contrib. Bot., 43:1-62.

Soulé, ME \& BA Wilcox. 1980. Conservation Biology. An evolutionary-ecological perspective. Sinauer Associates, Inc. Sunderland, Massachusetts.

South, DB; SW Harris; JP BarnetT; MJ Hainds \& DH GJERSTAD. 2005. Effect of container type and seedling size on survival and early height growth of Pinus palustris. For. Ecol. Manage., 204:385-398.

Trabucco, A \& RJ Zomer. 2009. Global Aridity Index (Global-Aridity) and Global Potential Evapo-Transpiration (Global-PET) Geospatial Database. CGIAR Consortium for Spatial Information. Published online, available from the CGIAR-CSI GeoPortal at: www.cgiar-csi.org.

Verga, E; G Leynaud; J Lescano \& LM Bellis. 2012. Is livestock grazing compatible with the amphibian diversity of the highland Mountains of central Argentina? Europ. J. Wildl. Res., 58:823-832

Vivian, LM; GL Cary; RA Bradstock \& AM Gill. 2008. Influence of fire severity on the regeneration, recruitment and distribution of eucalypts in the Cotter River Catchment, Australian Capital Territory. Austral Ecol., 33:55-67.

Wright, SJ; GA SÁnChEZ-AzofeIfa; C Portillo-Quintero $\&$ D DAVIES. 2007. Poverty and corruption compromise tropical forest reserves. Ecol. Applic., 17:1259-1266.

Zimmermann, H; D Renison; I Leyer \& I Hensen. 2009. Do we need livestock grazing to promote Polylepis australis tree recruitment in the Central Argentinean Mountains? Ecol. Research., 24:1075-1081. 


\section{INFORMACIÓN SUPLEMENTARIA}

Apéndice 1. Lista bibliográfica actualizada a octubre de 2012 de trabajos publicados en revistas científicas, congresos, como tesis y que tratan sobre la ecología, manejo y conservación de bosque de Polylepis argentinos. En el caso de congresos y tesis se omitió la cita cuando la misma información se encontraba ya disponible en una publicación científica. La cita se encuentra en la primera columna, y las provincias donde se realizó la investigación en la segunda columna.

Acosta, A. 1986. Estructura poblacional de Polylepis australis. Pp. 392-401 en: Efecto de las actividades humanas sobre los ecosistemas montañosos y de tundra. MAB-PNUMA, Córdoba, Argentina.

Azocar, A; F RAda \& C GARcíA-NúÑEZ. 2007. Functional characteristics of the arborescent genus Polylepis along a latitudinal gradient in the high Andes. Interciencia, 32:663-668.

Bellis, LM; L Rivera; N Politi; E Martín; ML Perasso; et AL. 2009. Latitudinal patterns of bird richness, diversity and abundance in Polylepis australis mountain forest of Argentina. Bird Conserv. Intern., 19:265-276.

Bellis, LM; A Pidgeon; C Alcántara Concepción; L HeIl \& V RAdeloff. 2010. Bird responses to landscape heterogeneity in highland mountain forests of central Argentina. 25th International Ornithological Congress, Campos do Jordão, Brazil.

Braun Wilke, RH. 1988. Productividad ecológica de la "queñoa" (Polylepis tomentella) en la Puna de Jujuy. VI Congreso Forestal Argentino. Santiago del Estero, Argentina.

Braun Wilke, RH \& GF GuZMÁn. 2005. Caracterización Ambiental de Queñoales de la Puna Jujeña (NO Argentino). Agraria, 2:3-7.

Braun Wilke, RH; LPE Picchetti \& GF Guzmán. 2005. Desaparición de Queñoales de la Puna Jujeña (NO Argentino). Agraria, 2:73-77.

Cabido, M \& A Acosta. 1985. Estudio fitosociológico en bosques de Polylepis australis BITT. ("Tabaquillo") en las Sierras de Córdoba. Argentina. Documents Phytosociologiques, 9:385-400.

CANTERO, JJ \& CA BiAnCo. 1987. El límite austral de Polylepis australis Bitt. (Tabaquillo) en la República Argentina. Parodiana, 5:65-71.

Cingolani, AM; D Renison \& MR Cabido. 2004. Mapping vegetation in a heterogeneous mountain rangeland using Landsat data: an alternative method to define and classify land-cover units. Remote Sens. Environ., 92:84-97.

Christie, DA; A Lara; J Barichivich; R Villalba; MS MorALES; ET AL. 2009. El Niño-Southern Oscillation signal in the world's highest-elevation tree-ring chronologies from the Altiplano, Central Andes. Palaeogeography, Palaeoclimatology, Palaeoecology, 281:309-319.

Cingolani, AM; D Renison; P Tecco; DE Gurvich \& M CABIDO. 2008 Predicting cover types in a mountain range with long evolutionary grazing history: a GIS approach. J. Biogeogr., 35:538-551

ENRICO, L; G FunEs \& M CABIDO. 2004. Regeneration of Polylepis australis Bitt. in the mountains of central
Argentina. For. Ecol. Manage., 190:301-309.

FERNÁNDEZ, J. 1970. Polylepis tomentella y orogenia reciente. Boletín de la Sociedad Argentina de Botánica, 13:14-30.

García, C; D Renison; AM Cingolani \& E FernándezJurICIC. 2007. Avifaunal changes as a consequence of large-scale livestock exclusion in the mountains of Central Argentina. J. Appl. Ecol., 45:351-360.

GARcía, ME. 2006. Lluvia polínica en selvas montanas de la provincia de Tucumán, Argentina. Revista del Museo Argentino de Ciencias Naturales, 8:159-164.

GILMONTERO, R \& R VillalbA. 2005. Tree rings as a surrogate for economic stress? an example from the Puna of Jujuy, Argentina in the 19th century. Dendrochronologia, 22: 141-147.

Giorgis, M; AM Cingolani; I Teich; D Renison \& I Hensen. 2010. Do Polylepis australis trees tolerate herbivory? Seasonal patterns of shoot growth and its consumption by livestock. Plant Ecol., 207:307-319.

Giorgis, M; P Tecco; AM Cingolani; D Renison; P Marcora; ET AL. 2011. Factors associated with woody alien species distribution in a newly invaded mountain system of central Argentina. Biol. Invas., 13:1423-1434.

GuZMÁN, GF. 1997. In uencia del pastoreo en la regeneración del bosque de queñoa (Polylepis tomentella Wedd.) en la puna de Jujuy (Argentina). Tesis de Maestría en Conservación y Gestión del Medio Natural. Universidad Internacional de Andalucía-España.

Heil, L; E Fernández-Juricic; D Renison; AM Cingolani \& DT Blumstein. 2006. Avian responses to tourism in the biogeographically isolated high Córdoba Mountains, Argentina. Biodiv. Conserv., 16:1009-1026.

Hensen, I; I TeIch; H HiRsch; H von WeHRdEN \& D RENISON. 2011. Range-wide genetic structure and diversity of the endemic treeline species Polylepis australis (Rosaceae) in Argentina. Amer. J. Bot., 98:1825-1933.

Julio, N; A Sobral; J rondan Dueñas, J Dirienzo; D RENISON; ET AL. 2008. RAPD and ISSR markers indicate diminished gene flow due to recent fragmentation of Polylepis australis woodlands in central Argentina. Bioch. Syst. Ecol., 36:329-335.

Julio, NB; J Rondan Dueñas; D Renison \& I Hensen. 2011. Genetic structure and diversity of Polylepis australis (Rosaceae) tree populations from central Argentina: Implications for forest conservation. Silvae Genetica, 60:55-61.

KoptA, RF. 1985. Estudio sobre la germinación de Polylepis australis BITT., y algunas consideraciones preliminares sobre la diseminación y depredación de sus unidades dispersantes. Tesis de Grado para optar al título de Biólogo. Universidad Nacional de Córdoba, Córdoba, Argentina.

LANDi, M \& D Renison. 2010. Forestación con Polylepis australis en suelos erosionados de las Sierras Grandes de Córdoba: evaluación del uso de terrazas y vegetación nodriza. Ecol. Aust., 20:47-55.

LETT, I. 2006. Efectos de la fragmentación del bosque de Polylepis australis BITT. sobre la herbivoría causada por insectos y su incidencia en la viabilidad de las semillas. Tesis de Grado para optar al título de Biólogo. Universidad Nacional de Córdoba, Córdoba, Argentina.

Lupo, L; MR Morales; HD Yacobaccio; A Maldonado \& M Grosjean. 2007. Cambios ambientales en la Puna jujeña durante los últimos 1200 años: explorando su impacto en la economía pastoril. XVI Congreso Nacional de Arqueología Argentina. Tomo III. Pp. 151-.156. Ediciones Universidad Nacional de Jujuy.

Marcora, P; I Hensen; D Renison; P Seltmann \& K Wesche. 
2008. The performance of Polylepis australis trees along their entire altitudinal range: implications of climate change for their conservation. Diver. Distrib., 14:630636.

Martino, J; C Urcelay \& D Renison. 2011. Crecimiento y colonización micorrícica de Polylepis australis Bitter (Rosaceae) en suelos con distinta historia de pastoreo. Kurtziana, 36:69-77.

Menghi, M \& R Del Sueldo. 2007. Landscape mosaic, habitat structure and fragmentation of native forests at Córdoba mountain areas (Argentina central). En: Pacha, MJ; S Luque; L Galetto \& L Iverson (eds.). Understanding biodiversity loss: an overview of forest fragmentation in South America. IALE Landscape Research and Management papers. International Association of Landscape Ecology.

Menoyo, E; AG BeCERRa \& D Renison. 2007. Mycorrhizal associations in Polylepis woodlands of Central Argentina. Can. J. Bot., 85:526-531.

Menoyo, E; D Renison \& AG Becerra. 2009 Arbuscular mycorrhizas and performance of Polylepis australis trees in relation to livestock density. For. Ecol. Manage., 258: 2676-2682.

Morales, MS; R Villalba; HR Grau \& L Paolini. 2004. Rainfall-controlled tree growth in high-elevation subtropical treelines. Ecology, 85:3080-3089.

Perasso, ML. 2009. Uso de la percepción remota en la elaboración de un modelo predictivo de riqueza de aves en bosquecillos de Polylepis australis de las Sierras Grandes de Córdoba. Tesis de Grado para optar al título de Biólogo. Universidad Nacional de Córdoba, Córdoba, Argentina.

Picchetti, LP; GF Guzmán \& BS Villafañe. 2001. Dos nuevas especies del género Polylepis para la Argentina. Simposio sobre Ecología de Bosques de Polylepis. V CLAE. Universidad Nacional de Jujuy, S.S. de Jujuy, Argentina. Pp 769.

Picchetti, LP; BS Villafañe \& GF GuZmán. 2004. Ensayos de germinación en Polylepis spp. (Rosaceae), "queñoa", de rodales de la puna jujeña. Agraria, 1:71-75.

Pollice, J; P Marcora \& D Renison. 2012. Seed production in Polylepis australis (Rosaceae) as influenced by tree size, livestock and interannual climate variations in the mountains of central Argentina. New Forests, Published online.

Rajchenberg, M \& GL Robledo. 2005. Aleurocystis gloeocystidiata sp. nov. on Polylepis australis in Argentina. Mycotaxon, 92:317-322.

Renison, D \& AM Cingolani. 1998. Experiencias en germinación y reproducción vegetativa aplicados a la reforestación con Polylepis australis (Rosaceae) en las Sierras. AgriScientia, 15:47-53.

Renison, D \& AM Cingolani. 2002. Evaluación de la supervivencia y crecimiento de plantines de Polylepis australis (Rosaceae) para la elección de plantas semilleras. AgriScientia, 19:63-66.

Renison, D; AM Cingolani \& D SChInNER. 2002. Optimising restoration of Polylepis australis forests through reforestation: When, where and how to transplant seedlings to the mountains? Ecotropica, 8:219-224.

Renison, D; AM Cingolani \& R SuÁRez. 2002. Efectos del fuego sobre un bosquecillo de Polylepis australis (Rosaceae) en las montañas de Córdoba, Argentina. Rev. Chil. Hist. Nat., 75:719-727.

Renison, D; AM Cingolani; R Suárez; E Menoyo; C CoutsIERS; ET AL. 2005. The restoration of degraded mountain forests: effects of seed provenance and microsite characteristics on Polylepis australis seedling survival and growth in Central Argentina. Rest. Ecol., 13:129-137.

RenIsON, D;IHENSEN \& AMCINGOLANI. 2004. Anthropogenic soil degradation affects seed viability in Polylepis australis mountain forests of central Argentina. For. Ecol. Manage., 196:327-333.

Renison, D; I Hensen; R Suárez \& AM Cingolani. 2006. Cover and growth habit of Polylepis woodlands and shrublands in the mountains of central Argentina: human or environmental influence? J. Biogeogr., 33: 876-887.

Renison, D; I Hensen; R SuÁrez; AM Cingolani; P Marcora; ET AL. 2010. Soil conservation in Polylepis mountain forests of Central Argentina: Is livestock reducing our Natural capital? Austral Ecology, 35:435-443.

Renison, D; I Hensen \& R SuÁrez. 2011. Landscape Structural Complexity of High-Mountain Polylepis australis Forests: A New Aspect of Restoration Goals. Restor. Ecol., 19:390-398.

Robledo, G; C Urcelay \& M Rajchenberg. 2003. New species causing decay on living Polylepis australis in Cordoba, central Argentina. Mycologia, 95:347-53.

Robledo, G; C Urcelay; L Domínguez \& M Rajchenberg. 2006. Taxonomy, ecology and biogeography of Polypores (Basidiomycetes) from Argentinian Polylepis woodlands. Can. J. Bot., 84:1561-1572.

RoBLEDO, GL \& D RENISON. 2010. Wood-decaying polypores in the mountains of central Argentina in relation to Polylepis forest structure and altitude. Fungal Ecol., 3: 178-184.

SALVI, VF. 2007. El registro arqueobotánico en el sitio "Arroyo El Gaucho I" durante el Holoceno temprano (8000-6000 AP) (Pampa de Achala, Córdoba). Comechingonia Virtual, 1:1-11.

Seltmann, P; A Cocucci; D Renison; A Cierjacks \& I Hensen. 2009. Mating system, outcrossing distance effects and pollen availability in the wind-pollinated treeline species Polylepis australis BITT. (Rosaceae). Basic Appl. Ecol., 10:52-60.

Seltmann, P; I Hensen; D Renison; K Wesche; S Ploch; ET AL. 2009. Biparental inbreeding depression, genetic relatedness and progeny vigour in a wind-pollinated treeline species in Argentina. Plant Ecol., 205:155-164.

Seltmann, P; I Leyer; D Renison \& I Hensen. 2007. Variation of seed mass and its effects on germination in Polylepis australis: Implications for seed collection. New Forests, 33:171-181.

Seltmann, P; D Renison; A Cocucci; I Hensen \& K JUNG. 2007. Fragment size, pollination efficiency and reproductive success in natural populations of windpollinated Polylepis australis (Rosaceae) trees. Flora, 202: 547-554.

Solíz, C; R Villalba; J Argollo; MS Morales; D Christie; ET AL. 2009. Spatio-temporal variations in Polylepis tarapacana radial growth across the Bolivian Altiplano during the 20th century. Palaeog. Palaeocl. Palaeoecol., 281:296-308.

SuÁrez, ML; D Renison; P Marcora \& I Hensen. 2008. Agesize-habitat relationships for Polylepis australis: dealing with endangered forest ecosystems. Biodivers. Conserv., 17:2617-2625.

Teich, I; AM Cingolani; D Renison; I Hensen \& M Giorgis. 2005. Do domestic herbivores retard Polylepis australis Bitt. woodland recovery in the mountains of Córdoba, Argentina? For. Ecol. Manage., 219:229-241. 
Torres, R; D Renison; I HenSEn; R SuÁREZ \& L EnRICo. 2008. Polylepis australis' regeneration niche in relation to seed dispersal, site characteristics and livestock density. For. Ecol. Manage., 254:255-260.

Verga, E; G Leynaud; J Lescano \& LM Bellis. 2012. Is livestock grazing compatible with the amphibian diversity of the highland Mountains of central Argentina? Europ. J. Wildl. Res., 58:823-832.

Zimmermann, H; D Renison; I LeYer \& I Hensen. 2009. Do we need livestock grazing to promote Polylepis australis tree recruitment in the Central Argentinean Mountains? Ecol. Research., 24:1075-1081.

Apéndice 2. Áreas protegidas que albergan o potencialmente podrían albergar alguna de las especies de Polylepis en Argentina. Los códigos de especie representan: P. ta: P. tarapacana; P. to: P. tomentella; P. c-g: P. crista-galli; P. hi: $P$. hieronymi; $P$. au: $P$. australis. Cuando los mismos están entre paréntesis, indica área potencial para la especie sin confirmación de su presencia verdadera. Los códigos de categoría representan: PN: Parque Nacional; PP: Parque Provincial; RN: Reserva Nacional; RP: Reserva Provincial; RB: Reserva de la Biosfera; MN: Monumento Natural; RPriv: Reserva Privada, RNE: Reserva Natural Estricta. PH: Patrimonio de la Humanidad, BM: Bosque Modelo. La efectividad fue categorizada en alta, media y baja subjetivamente en base al conocimiento de los autores y ayudados por información del libro de Chebez (2005). Entre paréntesis luego de la efectividad si indica si el área protegida dispone de Instrumento Legal de creación óptimo (IL); Plan de Manejo (PM) y Personal a campo (PC) según (Chebez 2005).

\begin{tabular}{|c|c|c|c|c|c|c|}
\hline Nombre & Sp. de Polylepis & Categoría & Provincia & Tenencia & Área $\left(\mathrm{km}^{2}\right)$ & Efectividad \\
\hline Potrero de Yala & P. au (P.hi) & $\mathrm{PP}, \mathrm{RB}$ & Jujuy & Provincial & 16 & Baja (sin IL, PM y PC) \\
\hline Calilegua & P. au, hi, $(c-g)$ & PN, RB & Jujuy & Nacional & 736 & Alta (con IL, PM y PC) \\
\hline Yungas & P. au, hi,c-g & $\mathrm{RB}$ & Jujuy-Salta & Provincial & 14000 & Baja (con IL y PM, sin PC) \\
\hline Tesorero & $(P . a u, h i)$ & RPriv & Jujuy & Privada & 40 & - \\
\hline Portal de Piedra & P. hi & RPriv & Jujuy & Privada & 4 & - \\
\hline Cachipunco & (P. hi) & RPriv & Jujuy & Privada & 11 & - \\
\hline Laguna de los Pozuelos & P. to (P. ta) & RB y MN & Jujuy & Nacional & 145 & Baja (sin IL y PM, con PC) \\
\hline Queñoas de Mocaraite & P. to & RPriv & Jujuy & Privada & 4 & - \\
\hline Tinte & P. to & $\mathrm{RN}$ & Jujuy & Privada & 800 & - \\
\hline Quebrada de Humahuaca & P. au, P. hi, P.cri & $\mathrm{PH}$ & Jujuy & Provincial & 5200 & Baja (con IL, sin PM y PC) \\
\hline El Rey & P. hi & PN, PP y RNE & Salta & Nacional & 419 & Alta (conIL,sinPMyconPC) \\
\hline Baritú & P. au (hi) & PN y RNE & Salta & Nacional & 622 & Alta (con IL, sin PM, con PC) \\
\hline Nogalar & P. $a u,(h i, c-g)$ & RN y RB & Salta & Nacional & 32 & Media (sinILyPM, conPC) \\
\hline Finca Las Costas & P. au, P. hi & $\mathrm{RP}$ & Salta & Provincial & 86 & Baja (sin IL, PM y PC) \\
\hline Santa Ana & P. au (hi) & $\mathrm{RP}$ & Tucumán & Provincial & 200 & Media (sin IL, PM y PC) \\
\hline La Florida & P. au & $\mathrm{RP}$ & Tucumán & Provincial & 102 & Media (conIL,sin PMyPC) \\
\hline Los Alisos & P. au & PN & Tucumán & Nacional & 154 & Alta (conIL, sin PM, conPC) \\
\hline Quebrada del Portugués & P. au & RP y RNE & Tucumán & Nacional & 160 & Baja (sin IL, PM y PC) \\
\hline Cumbres Calchaquíes & P. au & $\mathrm{RP}$ & Tucumán & Nacional & 794 & Media (conIL,sinPMyPC) \\
\hline Bosque Modelo & P. au, P. hi & $\mathrm{BM}$ & Tucumán & Provincial & 1800 & - \\
\hline Quebrada del Condorito & P. au & PN y RN & Córdoba & Nacional & 260 y 120 & Alta (con IL, PM y PC) \\
\hline Hídrica de Achala & P. au & $\mathrm{RP}$ & Córdoba & Privada & 1170 & Media (con IL, PM y PC) \\
\hline Tabaquillo & P. au & RPriv & San Luis & Privada & 2,5 & - \\
\hline
\end{tabular}

